\title{
Development of a Method for Optimizing Rotating Machines Vibrations Limits
}

BENDJAIMA Belkacem

M'sila, Algérie

Abstract: This work consists to a development a new calculation method of rotating machines vibration limits. we will confirmed norms. An evaluation of the absolute vibration parameters of these machines with a comparison of vibration limits of ISO 10816-1 will be also affected.

Key words: Vibration, stiffness, centrifugal force, free movement.

\section{Introduction}

Rotating machines operate with limits vibrations, an update of these limits maybe required.

From the following calculation we will try to reformulate terms of vibrations machine parameters.

\section{Model of the Machines Vibrations [1]:}

The rotating machines vibrations will be modeled by the system with one degree on Fig. 1. Rotating machine can be compared with this system (Fig. 2):

- The base is replaced by the bearings.

- The Springs is replaced by the rotor.

- The mass is replaced by the body of the turbine or the pump.

- Damping is ensured by the fluid passing in the turbine or the pump.

\section{Basic Equation[1]}

The differential equation translating the behavior of the system (displacement of mass $\mathrm{x}(\mathrm{t})$ compared to its rest position after release) in a direction can be written:

$$
M \ddot{x}(t)+C \dot{x}(t)+k x(t)=F(t)
$$

With $\mathrm{k}$ is the rigidity of the spring which expresses

Corresponding author: BENDJAIMA Belkacem, Ph.D., research fields: turbomachinery performances and design, vibrations analysis and diagnostic. that the force of recall is proportional to elongation $\mathrm{x}$ and $\mathrm{F}$ the force applied on the basis.

A viscous damping (or fluid) of coefficient $C$ exerts on the movement of the mass $\mathrm{m}$ a force of damping $-C \frac{d x}{d t}$ proportional to the instantaneous speed. The total response of the movement is:

$$
x(t)=x_{L}(t)+x_{F}(t)
$$

where, $x_{L}(t)$ is the response of the free movement, solution of the equation:

$$
M \ddot{x}(t)+C \dot{x}(t)+k x(t)=0
$$

where, $x_{F}(t)$ is the response of the movement forced, solution of the equation:

$$
M \ddot{x}(t)+C \dot{x}(t)+k x(t)=F(t)
$$

The response of the free movement [1] in the case $\xi<1$ is:

$$
x_{L}(t)=X_{0} e^{-\xi \omega_{0} t} \sin \left(\omega_{0} \sqrt{1-\xi^{2}} t+\phi_{0}\right)
$$

where, $\xi=\frac{C}{C_{C}}$ and $C_{C}$ are critically damping.

The natural frequency of the free movement is $\omega_{0}=\sqrt{\frac{k}{M}}$. If $\xi=1$, then $C=2 \sqrt{k M}=2 M \omega_{0}=C_{C}$. These cases are rare, and when $\xi>1$, the free response is an exponential function. $x_{L}(t)$ is represented on the Fig. 3.

By holding account that $F(t)=F_{0} e^{j \omega t}$ the response of the forced movement is: $x(t)=X e^{j(\Omega t-\phi)}$, with: 


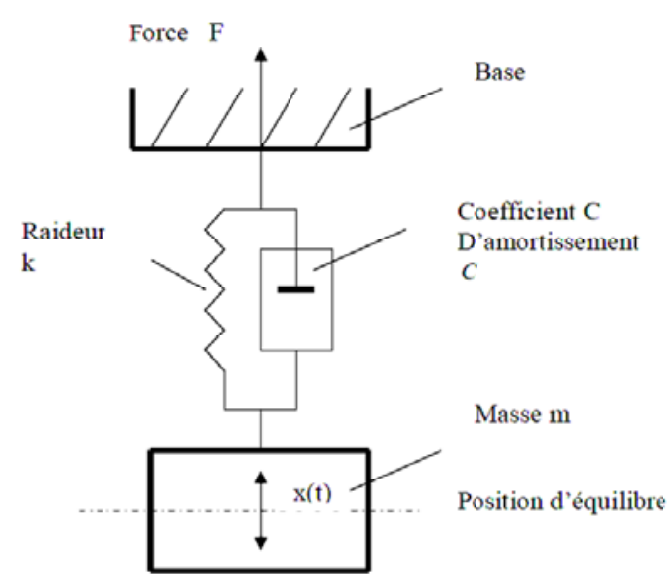

Fig. 1 mechanical system with one degree.

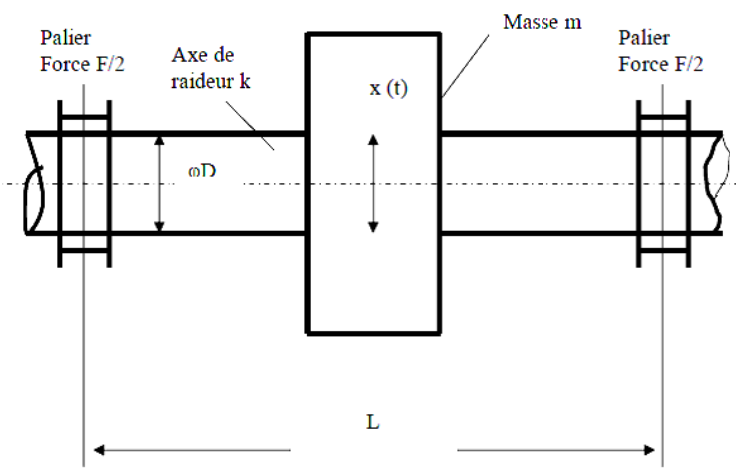

Fig. 2 Rotating machines system.

$$
\begin{gathered}
X=\frac{F_{0} / k}{\left[\left(1-\left(\frac{\Omega}{\omega_{0}}\right)^{2}\right)^{2}+\left(2 \xi \frac{\Omega}{\omega_{0}}\right)^{2}\right]^{1 / 2}} \\
\operatorname{tg} \phi=\frac{2 \xi \frac{\Omega}{\omega_{0}}}{1-\left(\frac{\Omega}{\omega_{0}}\right)^{2}} \\
T=\frac{2 \pi}{\omega_{0} \sqrt{1-\varepsilon^{2}}}
\end{gathered}
$$

If $\varepsilon \ll 1$, then $T=\frac{2 \pi}{\omega_{0}}$. With the natural pulsation $\omega_{0}=\sqrt{\frac{k}{M}}$. We can have the harmonic response forced to one degree on Fig. 4.

\section{Stiffness Calculation of Rotors}

At passage of the critical speed [2]:

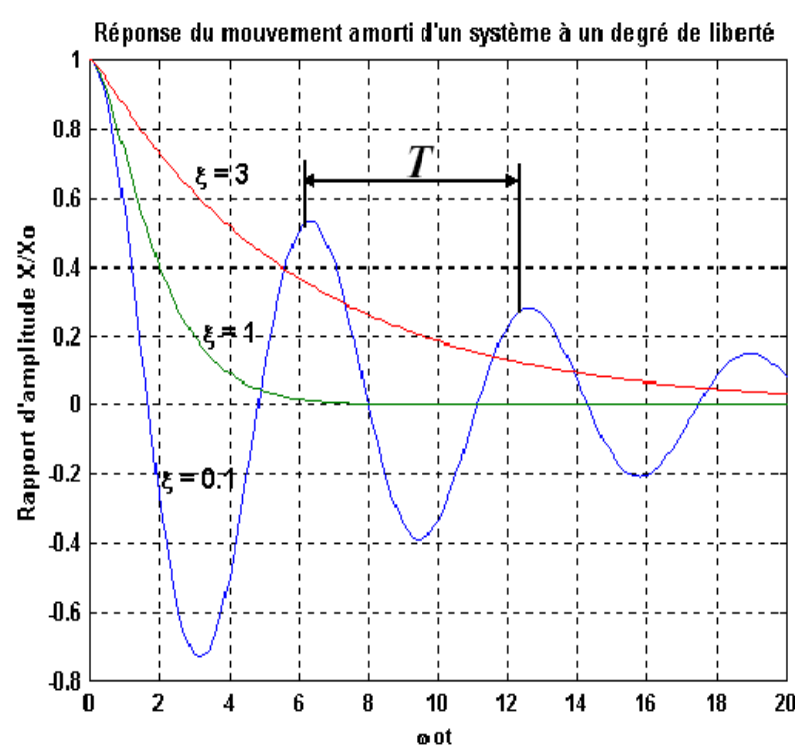

Fig. 3 Mechanical system with one degree.

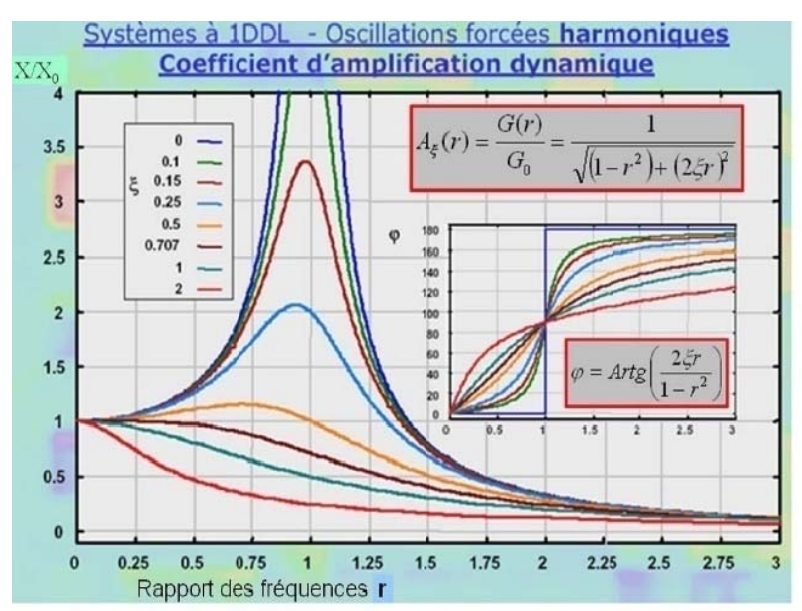

Fig. 4 Harmonic Forced response with one Degree.

$$
\begin{array}{r}
\Omega=\omega_{0}=\sqrt{\frac{k}{M}} \\
k=M \omega_{0}^{2}
\end{array}
$$

where, $\omega_{0}$ is the natural frequency corresponding to the critical speed, $k$ is rotor stiffness, $M$ is rotor mass. The equilibrium is realized. If $F_{b}=F_{c}$ (equality of centrifugal forces of unbalance and unbalance correction force with masse $\mathrm{m}$, then:

$$
M e \omega_{0}^{2}=m R \omega_{0}^{2}
$$

With e the excentricity and $\mathrm{R}$ rotor radius (distance from the position of the balancing mass to the rotor 
geometric center).We can write to this effect:

$$
m=\frac{M e}{R}
$$

On the other hand, the restoring force is given by:

$$
F=k \cdot x
$$

The expression of the stiffness (the restoring force per unit elongation) is given by:

$$
k=\frac{m R}{e} \cdot \omega_{0}^{2}
$$

We suppose that $x_{1}$ is vibrations amplitude at critical speed and $x_{2}$ is at nominal speed. We pose

$$
\begin{gathered}
\tau=\frac{x_{2}}{x_{1}}=\frac{x_{1}}{x_{0}} \\
\tau>1
\end{gathered}
$$

We suppose that $\tau$ is vibration ratio of $x_{2}$ and $x_{1}$ and also the ratio of still admissible $X_{1}$ vibration and admissible vibration $X_{0}$ according norm 10816-1 (Cf. [1]). We pose :

$$
\begin{gathered}
\omega_{1}=\rho \cdot \omega_{0} \\
\omega_{1}>\omega_{0}
\end{gathered}
$$

Here, $\omega_{1}$ and $\omega_{0}$ are nominal and critical speed.

\section{Balancing Quality Vibrations Limits Evaluation}

\subsection{Vibrations Expressions}

The tolerated residual unbalance is defined by norms from the balancing quality. Quality factor $G$ $(\mathrm{mm} / \mathrm{s})$ is defined by:

$$
G=e \cdot \omega_{1}
$$

where, $e$ is excentricity. $\omega_{1}$ is maximal angular speed. The stiffness is:

$$
k=\frac{m R}{G} \cdot \omega_{0}^{2} \cdot \omega_{1}
$$

$$
k=\frac{F_{C}}{G \cdot \rho} \cdot \omega_{0}
$$

With the centrifugal forces of the mass $m$ given by:

$$
F_{C}=m R \omega_{1}^{2}
$$

Then:

$$
\begin{aligned}
k & =C_{1} \cdot \omega_{0} \\
C_{1} & =\frac{F_{C}}{G \cdot \rho}
\end{aligned}
$$

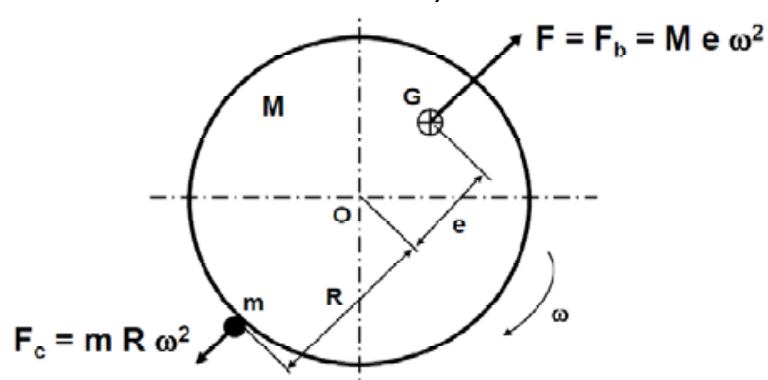

Fig. 5 Disposition on a rotor unbalance [2].

$$
\begin{gathered}
k=M \omega_{0}^{2} \\
C_{1}=M \cdot \omega_{0}
\end{gathered}
$$

The factor $C_{1}$ depends to centrifugal force at rotation nominal speed and to the naturalfrequency. For low damping:(cf. [1]) if:

$$
\tau=\frac{X_{1}}{X_{0}}=\frac{M}{k}
$$

With Eq. (3.2), we have:

$$
\begin{array}{r}
k=C_{1} \cdot \omega_{0}=\frac{M}{\tau} \\
C_{1}=\frac{M}{\tau \omega_{0}}
\end{array}
$$

Centrifugal force [3] is:

$$
F_{C}=m R \omega_{1}^{2}=\frac{\rho G M}{\tau \omega_{0}}
$$

Unbalance with the mass $m$ is given by:

$$
b=m R
$$

Then:

$$
b=\frac{G}{\tau} \cdot \frac{M}{\omega_{0}^{2}} \cdot \frac{1}{\omega_{1}}
$$


Unbalance is function of ratio rotor mass and rotating frequency. We have:

$$
b=\frac{G}{\tau} \cdot \frac{M}{\omega_{0}^{2}} \cdot \frac{T}{2 \pi}
$$

In case of a rotating machines:(case of excitation by a static unbalance, rigid rotor) we have the liaison on Fig. 6. With e like eccentricity, $\mathrm{C}$ center of gravity et $\mathrm{G}$ is inertia center, $\mathrm{z}(\mathrm{t})$ and $\mathrm{y}(\mathrm{t})$ are the co-ordinates of the center of gravity along two perpendicular directions. The equations of the movement are:

$$
m \frac{d^{3}}{d t^{2}}(y(t)+e \cos (\Omega t))=-k y(t)-c y(t)
$$

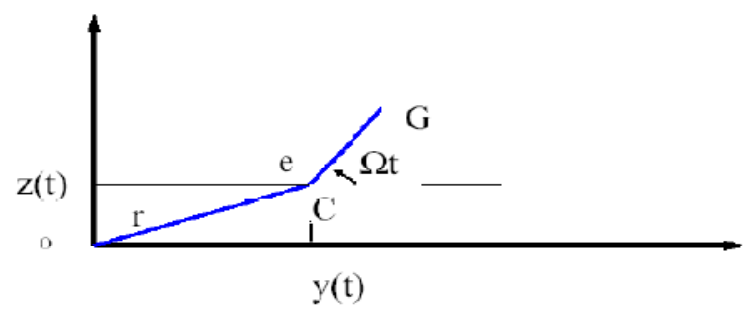

Fig. 6 Rigid liaison.

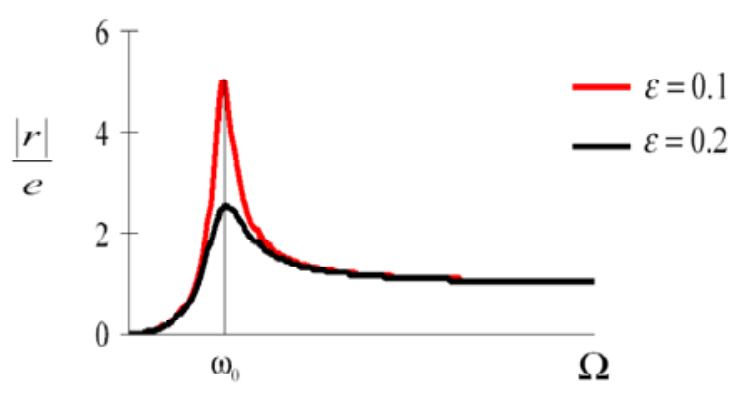

Fig. 7 Critical speed.

$$
m \frac{d^{3}}{d t^{2}}(z(t)+e \sin (\Omega t))=-k z(t)-c \dot{z}(t)
$$

With $\quad r(t)=y(t)+i z(t) \quad, \quad$ thus $m \ddot{r}(t)+c \dot{r}(t)+k r(t)=m e \Omega^{2} e^{j \Omega t}$. We have the solution:

$$
r(t)=\frac{e\left(\frac{\Omega}{\omega_{0}}\right)^{2}}{\left(1-\left(\frac{\Omega}{\omega_{2}}\right)^{2}\right)+j\left(2 \varepsilon \frac{\Omega}{\omega_{0}}\right)} e^{j(\Omega t-\varphi)}(4.12)
$$

For $\Omega=\omega_{0}$, that corresponds to critical speed (Fig. 7). The expression of the vibrations in the case of rotating machines and for nominal speed of rotation $\Omega_{1}$, we can write (cf. [1]):

$$
\frac{r_{1}}{e_{1}}=\frac{\left(\frac{\Omega_{1}}{\omega_{0}}\right)^{2}}{\left[\left(1-\left(\frac{\Omega_{1}}{\omega_{0}}\right)^{2}\right)^{2}+\left(2 \varepsilon \frac{\Omega_{1}}{\omega_{0}}\right)^{2}\right]^{1 / 2}}
$$

In the case of a constant speed $\Omega_{1}$, we will have:

$$
\frac{r_{1}}{e_{1}}=\frac{c}{k}
$$

With low damping $(\varepsilon \ll 1)$ :

$$
\frac{r_{1}}{e_{1}}=\frac{M}{k}
$$

With Eq. (4.11) and for one rotation:

$$
b=M \cdot e
$$

or

$$
e=\frac{1}{2 \pi} \cdot \frac{G}{\tau} \cdot \frac{1}{\omega_{0}^{2}}
$$

We have (cf. [1]) for rotating machines the ratio:

$$
\tau=\frac{r_{1}}{e}
$$

then:

$$
r=\frac{G}{2 \pi} \cdot \frac{1}{\omega_{0}^{2}}
$$

with

$$
r(t)=x(t)+i z(t)
$$

We have:

$$
x(t)=\frac{G}{2 \pi} \cdot \frac{1}{\omega_{0}^{2}}
$$

and (Cf.[1]):

$$
\begin{gathered}
x_{1}=B_{2}(\omega) \cdot \frac{M}{k}=B_{2}(\omega) \cdot \tau \\
x_{2}=B_{2}(\omega) \cdot \tau^{2}
\end{gathered}
$$

When we pose:

the value of $x_{1}$ is:

$$
\tau=\frac{M}{k}
$$

$$
x_{1}=\frac{G}{2 \pi} \cdot \frac{1}{\omega_{0}^{2}}
$$

then

$$
B_{2}(\omega)=\frac{1}{\tau} \cdot \frac{G}{2 \pi} \cdot \frac{1}{\omega_{0}^{2}}
$$




$$
x_{2}=\tau \cdot \frac{G}{2 \pi} \cdot \frac{1}{\omega_{0}^{2}}
$$

If we want to change $x_{2}$ we increases $\tau$. These values are constants and depends to critical speed. The ratio of vibration at speed $\Omega_{1}=\omega_{1}$ is :(cf. [1]):

$$
\frac{x_{2}}{x_{1}}=\frac{1}{1-\left(\frac{\Omega_{1}}{\omega_{0}}\right)^{2}}
$$

We can write :

$$
x_{2}=\frac{G}{2 \pi} \cdot \frac{1}{\omega_{0}^{2}} \cdot \frac{1}{1-\left(\frac{\Omega_{1}}{\omega_{0}}\right)^{2}}
$$

If $\Omega_{1}=\omega_{0}$, then $x_{2}$ tends to the infinite,

$$
x_{2}=\frac{G}{2 \pi} \times \frac{1}{\omega_{0}^{2}-\Omega_{1}^{2}}
$$

vibrations amplitudes is positive quantity. The expressions obtained are for condition:

$$
\Omega_{1}<\omega_{0}
$$

If

$$
\Omega_{1}>\omega_{0}
$$

Then:

$$
x_{2}=\frac{G}{2 \pi} \cdot \frac{1}{\omega_{0}^{2}+j \Omega_{1}^{2}}
$$

We have:

$$
\begin{gathered}
2 \pi\left(\omega_{0}^{2}+j \Omega_{1}^{2}\right) \cdot x_{2}=G \\
x_{2}=\frac{G}{2 \pi \omega_{0}^{2}}=x_{1}
\end{gathered}
$$

Or $x_{2}=0$, in case where (for example a generator or gas turbine):

$$
\Omega_{1}>\omega_{0}
$$

At nominal speed, we have: $x_{2}=x_{2}=\frac{G}{2 \pi \omega_{0}^{2}}$ and $\tau=1$

\subsection{Vibrations Limits}

For a movement applied to the base of the spring mass system (Fig. 1), with the form:

$$
Y(t)=Y_{0} \sin (2 \pi f t)
$$

We have (Cf. [4]):

$$
Y(t)=\frac{X}{Y} \sin (2 \pi f t+\Phi)
$$

and with the differential equation of movement: (Cf. [4]).

$$
\frac{X}{Y}(\omega)=\frac{1}{1+j\left(\frac{1}{Q}\right) \cdot\left(\frac{\omega}{\omega_{0}}\right)-\left(\frac{\omega}{\omega_{0}}\right)^{2}}
$$

where, $Q=\frac{1}{c} \sqrt{m \cdot k}$. The module is:

$$
\left|\frac{X}{Y}\right|=\frac{1}{\sqrt{1+\left(\frac{f}{f_{0}}\right)^{4}}-\left(2-\frac{1}{Q^{2}}\right)\left(\frac{f}{f_{0}}\right)^{2}}
$$

the vibration (velocity) is:

$$
v(t)=\frac{d x}{d t}=2 \pi f\left|\frac{X}{Y}\right| \cos (2 \pi f+\Phi)
$$

and the transfer functions:(Cf. [4])

$$
\frac{V}{Y}(\omega)=\frac{j \omega}{1+j\left(\frac{1}{Q}\right) \cdot\left(\frac{\omega}{\omega_{0}}\right)-\left(\frac{\omega}{\omega_{0}}\right)^{2}}
$$

For low damping, the module is:

$$
\left|\frac{V}{Y}\right|=\frac{\Omega_{1}}{\sqrt{1+\left(\frac{\Omega_{1}}{\omega_{0}}\right)^{4}-2\left(\frac{\Omega_{1}}{\omega_{0}}\right)^{2}}}
$$

where, $f$ is vibration frequency and $f_{0}$ is natural frequency.

We have:

$$
\frac{V}{X}=j \omega
$$

where, $V$ is velocity vibration and $X$ is vibration of the mass of spring mass system. The module is:

$$
|V|=|X|(2 \pi f)=x_{1}(2 \pi f)
$$

$$
V=\frac{\left(\frac{G}{2 \pi} \times \frac{\Omega_{1}}{\omega_{0}^{2}}\right)}{\sqrt{1+\left(\frac{\Omega_{1}}{\omega_{0}}\right)^{4}-2\left(\frac{\Omega_{1}}{\omega_{0}}\right)^{2}}}
$$

For our machine:

$$
V=\frac{\frac{G}{2 \pi} \cdot \frac{\rho}{\omega_{0}}}{\sqrt{1+\rho^{4}-2 \rho^{2}}}
$$

Vibration is function of frequency ratio. 
Table Nº1 Admissibles vibrations according norm ISO 10816-1.

\begin{tabular}{lllll}
\hline Group & Group $\mathrm{N}^{\circ} 01$ & Group $\mathrm{N}^{\circ} 02$ & Group $\mathrm{N}^{\circ} 03$ & Group $^{\circ} 04$ \\
\hline $\begin{array}{l}\text { Admissible vibration } \\
(\mathrm{mm} / \mathrm{s})\end{array}$ & 0.71 & 1.12 & 1.8 & 2.8 \\
$\begin{array}{l}\text { Still admissible Vibration } \\
(\mathrm{mm} / \mathrm{s})\end{array}$ & 4.5 & 7.1 & 11.2 & 18 \\
Coefficient $\boldsymbol{\tau}$ & 6.33 & 6.33 & 6.33 & 6.33 \\
\hline
\end{tabular}

\section{Case of Multiple Degrees of Freedom}

Free response is :

$$
x_{1}=X_{0}=B_{2}(\omega) \cdot \frac{M}{k}
$$

where, $X_{0}=\frac{1}{\tau} \cdot \frac{G}{2 \pi} \cdot \frac{1}{\omega_{0}^{2}} \cdot \frac{M}{k}$. We pose:

$$
\begin{gathered}
r=\frac{1}{\tau} \cdot \frac{G}{2 \pi}=\text { Constante } 1+\left(\frac{\Omega_{1}}{\omega_{0}}\right)^{4}-2\left(\frac{\Omega_{1}}{\omega_{0}}\right)^{2} \\
X_{0}=\frac{r}{\omega_{0}^{2}} \cdot[M] \cdot[k]^{-1}
\end{gathered}
$$

for multiple degrees of freedom:

$$
\left\{X_{0}\right\}=\frac{r}{\omega_{0}^{2}} \cdot[M] \cdot[k]^{-1}
$$

\section{Applications}

The following application is concerning a generator of IV group of machines which produces electrical energy. The generator is driven by a gas turbine.

Critical speed: at $50 \%$ of nominale speed.

Nominal speed: 3,000 rpm.

Speed ratio $: \rho=2$

For gas turbine: $\mathrm{G}=2.5 \mathrm{~mm} / \mathrm{s}$.

Maximale vibration is (see Eq. (4.42)):

$$
V=1.69 \mathrm{~mm} / \mathrm{s}
$$

RMS value is:

$$
V_{R M S}=\frac{V}{\sqrt{2}}=1.20 \mathrm{~mm} / \mathrm{s}
$$

According norm ISO 10816-1 the ratio $\tau$ is for all groups machines :

$$
\tau=\frac{\text { Vibrations }}{\text { Admissibles vibrations }}
$$

where, for admissibles vibrations: $\tau=1$; For still admissibles vibrations: $\tau=6.33$; For dangerous vibrations: $\tau>6.33$. Absolute vibrations (Cf. [1]) depend on $\tau$.

The optimal choice of this coefficient can change the thresholds and which takes into account machines design. If $\tau=\frac{X_{1}}{X_{0}}$, and considering that admissibles vibrations correspond to the balance, so we add them to the still admissible value. If $V_{1}$ correspond to the norm and $V_{2}$ correspond to searched vibration, we have:

$$
V_{2}=V_{1}-V_{1} \cdot \frac{\Delta V}{V}
$$

We calculate the relative difference $\frac{\Delta V}{V}$ between the value of the ISO 10816-1 norm and the calculated value of the machines group No. 04:

Table No2 1st Evaluation.

\begin{tabular}{lllll}
\hline Group & Group $\mathrm{N}^{\circ} 01$ & Group $\mathrm{N}^{\circ} 02$ & Group $\mathrm{N}^{\circ} 03$ & Group $^{\circ} 04$ \\
\hline $\begin{array}{l}\text { Admissibles vibrations } \\
(\tau=1)\end{array}$ & 0.31 & 0.48 & 0.77 & 1.20 \\
$\begin{array}{l}\text { Stilladmissibles vibrations } \\
(\tau=6.33)\end{array}$ & 1.93 & 3.05 & 4.9 & 7.62 \\
$\begin{array}{l}\text { Inadmissibles vibrations } \\
\tau>6.33\end{array}$ & $>1.93$ & $>3.05$ & $>4.9$ & $>7.62$ \\
\hline
\end{tabular}

Table $\mathrm{N}^{\circ} 03$ 2nd Evaluation.

\begin{tabular}{lllll}
\hline Groupe & Group $\mathrm{N}^{\circ} 01$ & Group No2 & Group No03 & Group No04 $^{\circ}$ \\
\hline Admissibles vibrations & 0.61 & 0.96 & 1.54 & 2.41 \\
Stilladmissibles vibrations & 2.54 & 4.12 & 6.45 & 10.03 \\
Inadmissibles vibrations & $>2.54$ & $>4.12$ & $>6.45$ & $>10.03$ \\
\hline
\end{tabular}


Table $N^{\circ} 04$ 3rd Evaluation.

\begin{tabular}{|c|c|c|c|c|}
\hline Group & Group $\mathrm{N}^{\circ} 01$ & Group $\mathrm{N}^{\circ} 02$ & Group $N^{\circ} 03$ & Group $\mathrm{N}^{\circ} 04$ \\
\hline Admissibles vibrations & 0.61 & 0.96 & 1.54 & 2.41 \\
\hline Stilladmissibles vibrations & 3.15 & 4.97 & 7.99 & 12.43 \\
\hline Inadmissibles vibrations & $>3.15$ & $>4.97$ & $>7.99$ & $>12.43$ \\
\hline \multicolumn{5}{|c|}{ Table $N^{\circ} 05 \quad 4$ th Evaluation. } \\
\hline Group & Group $\mathrm{N}^{\circ} 01$ & Group $\mathrm{N}^{\circ} 02$ & Group $\mathrm{N}^{\circ} 03$ & Group $\mathrm{N}^{\circ} 04$ \\
\hline Admissibles vibrations & 0.61 & 0.96 & 1.54 & 2.41 \\
\hline Stilladmissibles vibrations & 3.76 & 5.94 & 9.54 & 14.85 \\
\hline Inadmissibles vibrations & $>3.76$ & $>5.94$ & $>9.54$ & $>14.85$ \\
\hline
\end{tabular}

Table $N^{\circ} 6$ 5th Evaluation (corresponding to norm ISO 10816-1).

\begin{tabular}{lllll}
\hline Groupe & Group $\mathrm{N}^{\circ} 01$ & Group $\mathrm{N}^{\circ} 02$ & Group $\mathrm{N}^{\circ} 03$ & Group N $^{\circ} 04$ \\
\hline Stilladmissibles vibrations & 5.21 & 8.22 & 13 & 20.8 \\
\hline
\end{tabular}

Table $N^{\circ} 07 \quad 6$ th Evaluation.

\begin{tabular}{lllll}
\hline Group & Group $\mathrm{N}^{\circ} 01$ & Group N $\mathrm{N}^{\circ} 02$ & Group N 03 & ${\text { Group } \mathrm{N}^{\circ} 04}^{\circ}$ \\
\hline Admissibles vibrations & 0.61 & 0.96 & 1.54 & 2.41 \\
Stilladmissibles vibrations & 4.37 & 6.90 & 11.10 & 17.25 \\
Inadmissibles vibrations & $>4.37$ & $>6.90$ & $>11.10$ & $>17.25$ \\
\hline
\end{tabular}

Table $N^{\circ} 08$ Vibrations limitsfromoptmization (Cf. [1]).

\begin{tabular}{lllll}
\hline Group & Group N $\mathrm{N}^{\circ} 01$ & Group No2 & Group No3 & Group No04 $^{\circ}$ 02 \\
\hline Admissibles vibrations & 0.61 & 0.96 & 1.54 & 2.41 \\
Stilladmissibles vibrations & 4.98 & 7.86 & 12.63 & 19.66 \\
Inadmissibles vibrations & $>4.98$ & $>7.86$ & $>12.63$ & $>19.66$ \\
\hline
\end{tabular}

$$
\frac{\Delta V}{V}=\frac{2.8-1.2}{2.8}=0.57
$$

We construct the Table $\mathrm{N}^{\circ} 02$. We suppose also that admissibles vibrations corresponds to equilibirium, they can be added to still admissibles values. We have the five (05) evaluations to obtain vibrations values of norms (Cf. [1]). We also do the same operation on admissible and on still admissible values (Cf. Annexe). Vibrations from optmization (Cf.[1]) are identical to that calculated in the present document.

\subsection{Evaluations Number}

Considering $V_{E A-n}$ is still admissible vibration after $n$ evaluations, $V_{E A-1}$ is still admissible vibrations of the first evaluation and $V_{A}$ is admissibles vibrations, we have:

$$
V_{E A-n}=V_{E A-1}+n V_{A}
$$

the evaluations number is:

$$
\begin{gathered}
n=\frac{V_{E A-n}-V_{E A-1}}{V_{A}} \\
n=\left(18-\left(\frac{18}{6.33}\right)\right) / 2.8 \approx 5
\end{gathered}
$$

\section{Conclusions}

Our work is based on the optimization study of rotating machines vibration limits (see [1]). Depending on the coefficient as the ratio of vibrations and admissible vibrations according norm ISO 10816-1, absolutes vibrations limits origin is elaborated. We hope that this document will be the object of scientific research in the field of rotating machines.

\section{References}

[1] Bendjaima Belkacem. 2015. "Optimization of Rotating Machines Vibrations Limits by the Spring- Mass System Analysis." Journal of Materials Science and Engineering B 7-8: 323-30. 
[2] Conditional Maintenance by Vibration Analysis. 2010. Thermal Power plant of M' sila. Algeria.

[3] Boulenger, A. and Pachaud, C. 2007. "Aid Memory."
Analysis of Vibration Monitoring of Machines.

[4] Vibration Training. 1999. SCV Switzerland. Thermal Power Plant of M' sila. Algeria. 